\title{
Surface states and their possible role in the superconductivity of $\mathrm{MgB}_{2}$
}

\author{
V.D.P. Servedio ${ }^{1}$, S.-L. Drechsler ${ }^{2}$, T. Mishonov $^{3}$ \\ ${ }^{1}$ Institut für Oberflächenphysik und Mikrostrukturphysik, TU Dresden, D-01062 Dresden, Germany \\ ${ }^{2}$ Institut für Festkörper- und Werkstofforschung Dresden, D-01171 Dresden, Germany \\ ${ }^{3}$ LVSM, Katholieke Universiteit, Leuven, Belgium and University of Sofia, Bulgaria
}

\begin{abstract}
We report layer-Korringa-Kohn-Rostocker (LKKR) calculations for bulk and surface states (SFS) as well as the corresponding photoemission intensities of $\mathrm{MgB}_{2}$. Our theoretical results reproduce very well the recent angle resolved photoemission data by Uchiyama et al, cond-mat/0111152. At least two SFS are assigned. Consequences of SFS on the anisotropy of the upper critical fields and other properties in the superconducting state of grains in micro-powder samples are discussed.
\end{abstract}

PACS: 74.25.Jb, 74.70, 74.60.Ec, 73.-r

Recently, the first angle-resolved photoemission spectroscopy (ARPES) measurements on a $\mathrm{MgB}_{2}$ single crystal were reported by Uchiyama et al. [1]. They observed several dispersive states. Among them one has been ascribed to a surface state (SFS). The very existence of SFS and subsurface states for $\mathrm{MgB}_{2}$ as well as surface superconductivity have been predicted first in Refs. 2 and 3. However, there are significant quantitative differences between Ref. 2 and the ARPES data. The effects of SFS for the superconductivity in this challenging compound have not yet been discussed in detail. In particular, there is an unresolved puzzle with respect to the out-of plane anisotropy for the upper critical fields $\gamma_{H}=H_{c 2}^{a b} / H_{c 2}^{c}$. It is moderate for single crystals $1.6 \leq \gamma_{H} \leq 3.5$. [- 1 But large values $6 \leq \gamma_{H} \leq 9$ have been deduced indirectly from measurements on pure powder samples with small grains. [5] Enhanced superconductivity due to locally enhanced DOS (density of states) has been predicted [3] for idealized B- as well as Mg-terminated surfaces (BTS, MgTS) although in conflict with experimental data. We report on LKKR calculations for MgTS and BTS and compare them with the ARPES data. [1] Furthermore we propose a scenario which involves essentially SFS to resolve some puzzles of general interest mentioned above.

The electronic DOS for the semi-infinite system $\mathrm{MgB}_{2}$ as well as the photoemission spectra, were calculated using a LKKR code [8]. This code treats the photoemission process within the "one-step" approach. Dealing with the Green function formalism, it is capable to work with a complex potential, allowing the description of broadening effects due to the finite lifetime of quasi-particles. However, a set of input parameters must be provided phenomenologically in order to match experimental data as close as possible. As an input to the LKKR code, the real part of the one-electron effective complex potential was calculated self-consistently with the help of a LMTO (linear muffin-tin orbital) code. This preliminary calculation was done for the infinite $\mathrm{MgB}_{2}$ crystal with a basal plane lattice constant of $a=3.085 \AA$ and a ratio $c / a=1.142$. The von Barth-Hedin [9] correlation energy functional was used in connection with the local density approximation (LDA). The resulting Fermi energy $E_{F} \approx 13.1 \mathrm{eV}$ measured from the muffin-tin zero, while the muffin-tin radii were $1.711 \AA$ and $0.988 \AA$ for the $\mathrm{Mg}$ and $\mathrm{B}$, respectively. A direct evaluation of the self-energy correction to be added to the LMTO potential is notoriously very difficult. Hence, we fixed it empirically. As for its imaginary part we chose different values for states below and above the Fermi level, $-0.05 \mathrm{eV}$ and $-1 \mathrm{eV}$ respectively. Its real part was chosen to be $-1 \mathrm{eV}$ and applied to the photo-electron final states only. Rigorously, the full complex self-energy correction is not constant in the energy intervals under consideration, but here such details can be omitted without losing much accuracy.

The geometry of the semi-infinite $\mathrm{MgB}_{2}$ system was simply modeled cutting the ideal crystal perpendicularly to the $\hat{c}$ axis. Hence, no possible relaxation or reconstruction effects were taken into account. The topmost surface layer consists either of $\mathrm{Mg}$ atoms or B atoms. In the MgTS-case a work function $\phi=4.2 \mathrm{eV}$ was used, in the BTS $\phi=6.1 \mathrm{eV}$, as suggested in Ref. [2]. In both cases the surface potential barrier was modeled as a step potential, reflecting for occupied states and non reflecting, but refracting, for unoccupied ones. Its position $d$ was fixed to $0.987 \AA$ with respect to the topmost layer, while its height is given by the sum $E_{F}+\phi$. The energy of impinging photons at $28 \mathrm{eV}$ and the linear polarization $\perp$ to the symmetry directions $\Sigma$ and $\Lambda$ were chosen in accordance with the experiment. [1]

In Fig. 1 we show the DOS of the surface layer in

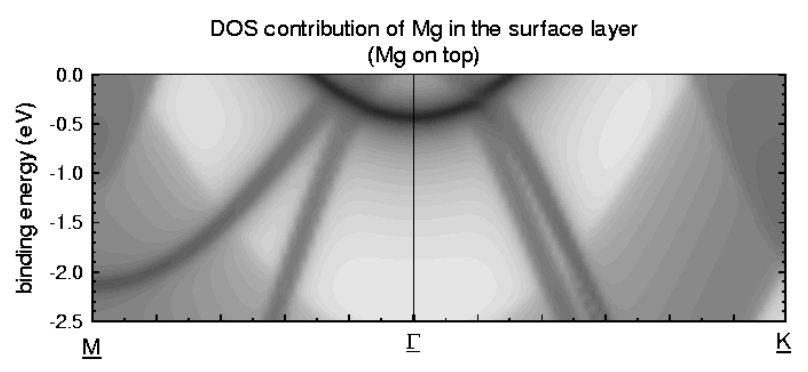

FIG. 1. The DOS in the topmost layer (MgTS-case) vs binding energy and $\mathbf{k}_{\|}$-vector along the $\overline{\boldsymbol{\Gamma}} \overline{\mathbf{M}}(\Sigma)$ and $\overline{\mathbf{\Gamma}} \overline{\mathbf{K}}(\Lambda)$ symmetry directions. The DOS is measured by the darkness on a logarithmic grey-scale. The step-like surface potential barrier is at a distance $d=0.99 \AA$ from the topmost layer. Notice the Mg-derived SFS at $\approx-0.5 \mathrm{eV}$ at $\overline{\boldsymbol{\Gamma}}$. 
the case of a MgTS. The quasi parabolic feature with a minimum around $-0.5 \mathrm{eV}$ on the $\overline{\boldsymbol{\Gamma}}$ point is clearly a SFS, lying in a gap in the $k_{z}$-projected bulk band structure [2]. It is $s, p_{z}, d_{z^{2}}$ in character, in a proportion roughly $3: 2: 1$ at the $\overline{\boldsymbol{\Gamma}}$ point. Its characteristic decay length is $2.6 \AA$, whereas the distance between the $\mathrm{Mg}$ and B planes is $1.762 \AA$. In Fig. 1, the dispersion of bulk bands is also visible. The narrower stripes correspond to the quasi 2-dimensional $\sigma$ bonded $\mathrm{p}_{x, y}$-bands, while the broad parabolic tape corresponds to the $\pi$ bonded $\mathrm{p}_{z}$-band.

SFS energies are much more sensitive to the values of the surface potential barrier positions $d$ than to the work functions $\phi$. Placing the barrier at a larger $d$ value results in a downward energy shift (cf. Figs. 2 and 3). Thus, the situation depicted in Ref. 2, where the energy of the SFS of a MgTS is $\approx-2 \mathrm{eV}$ at $\overline{\boldsymbol{\Gamma}}$, can be achieved with a surface potential barrier placed at $d=1.32 \AA$ above the topmost $\mathrm{Mg}$ layer. A different situation occurs in the BTS-case (cf. Fig. 3). Here the result of Ref. 2 is reproduced by a smaller $d$-value than our empirical one. This is probably due to the in-plane B-B distance which is $57 \%$ smaller than the $\mathrm{Mg}-\mathrm{Mg}$ one, so that in a BTS slab calculation, the image plane position could have been chosen nearer to the surface than in the MgTS. The SFS which we found for a BTS in the considered energy range, runs parallel to the $p_{z}$ broad band near its upper edge. It is depicted by the solid line peak at a binding energy $\approx-0.8 \mathrm{eV}$ in Fig. 3 calculated at $\mathbf{k}_{\|}=0.7 \cdot \overline{\mathbf{M}}$. It decays towards the bulk with a characteristic length of $9.8 \AA$ and exhibits $\mathrm{p}_{z}$ symmetry. If steps are present on the real surface, yielding islands of Mg- and BTS, a single effective

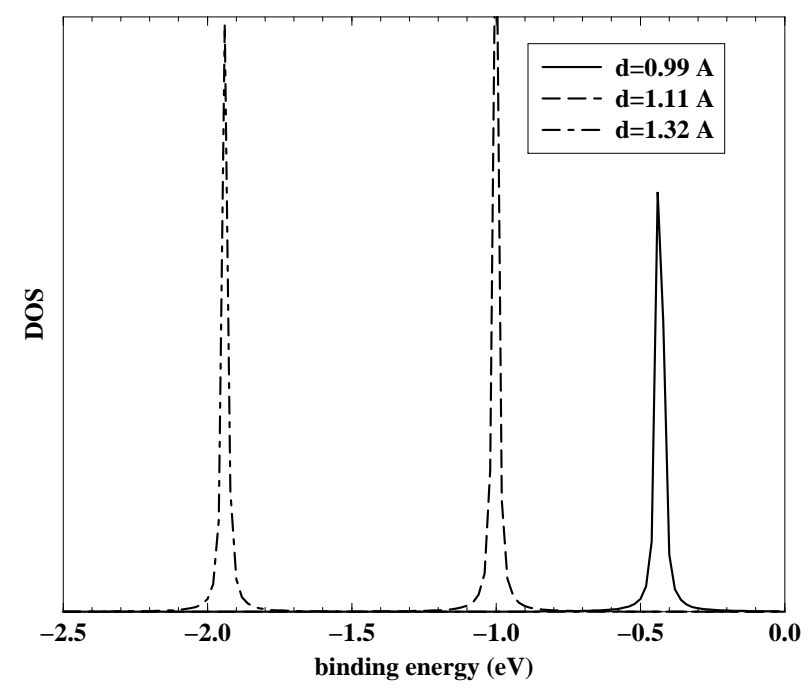

FIG. 2. The DOS of the surface layer in the case of a MgTS as a function of the binding energy, for $\mathbf{k}_{\|}=\overline{\boldsymbol{\Gamma}}$. The different curves were calculated with step-like surface potential barrier placed at various distances $d$ (given in $\AA$ ) from the surface. The imaginary part of the self-energy was set to $\operatorname{Im} \Sigma=-10 \mathrm{meV}$ in order to sharpen the peaks.

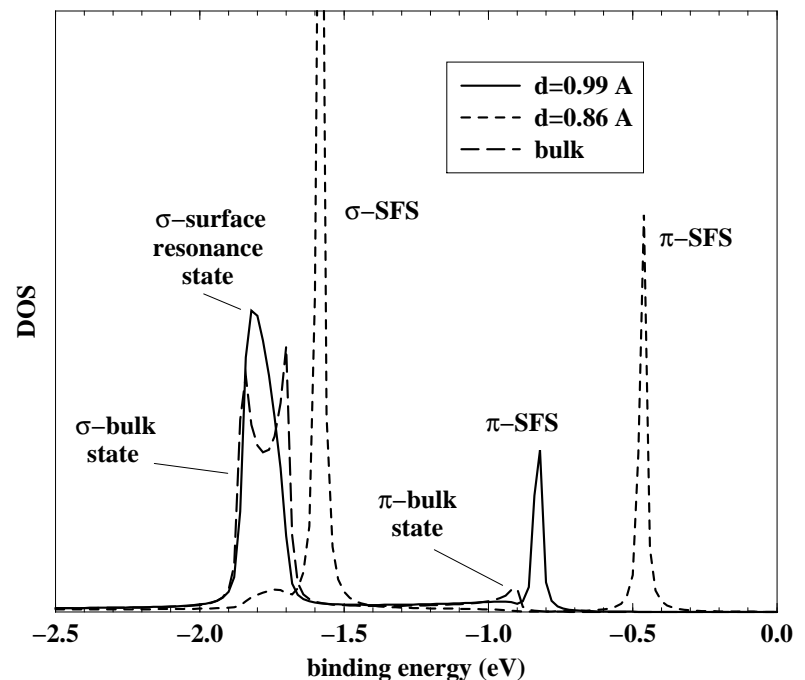

FIG. 3. The DOS of the surface layer in the BTS case as a function of the binding energy for $\mathbf{k}_{\|}=0.7 \cdot \overline{\mathbf{M}}$. The curves were calculated using a step-like surface potential barrier placed at different distances $d$ (given in $\AA$ ) from the topmost layer. $\operatorname{Im} \Sigma$ as in Fig. 2.

(averaged) $d$ value could be justified to analyze the ARPES data. In view to have either a MgTS a BTS (or even both if we have a step-like surface), we calculated the ARPES spectra shown in Figs. 4 and 6.

We would like to stress that the crucial effective $d$ parameter must be fitted from experimental data, and the only available data [1] is shown in Fig. 5 . Nice agreement with our calculations is obtained, if one superposes Figs. 4 and 6 , suggesting that the measured sample presented both Mg- and BTS. According to our calculations the feature centered at $\approx 0.4 \mathrm{eV}$ at $\overline{\boldsymbol{\Gamma}}$ is then to be assigned to a $\pi$-bonded $\mathrm{Mg}$ derived SFS in the case of MgTS. The parabolic feature centered at $\approx 2.7 \mathrm{eV}$ is most likely, in our opinion, to be assigned to a $\pi$-bonded B $2 p_{z^{-}}$ derived SFS arising from a BTS, in accordance with the calculations in Ref. 2. However, we admit that from the presented experimental data, an alternative assignment of this parabolic feature to the bulk B $2 \mathrm{p}_{z}$ derived $\pi$-band edge cannot be completely excluded. An analogous consideration holds for the $\mathrm{B} \sigma$ derived narrow bulk stripes, where a surface resonance state is found with our $d$ parameter value (cf. Fig. 3). Further ARPES experiments at variable photon energies should be performed to solve the full assignment problem. We emphasize that our interpretation mainly in terms of SFS does not reduce the importance of Ref. 1 which is the first experimental verification of the correct description of the electronic structure of $\mathrm{MgB}_{2}$ by the LDA. This is very important with respect to some times discussed strong many-body effects (correlation or non-adiabaticity) which would cause [10,11]) strong renormalizations of bulk and SFS as well. If strong correlation effects were present, they should 


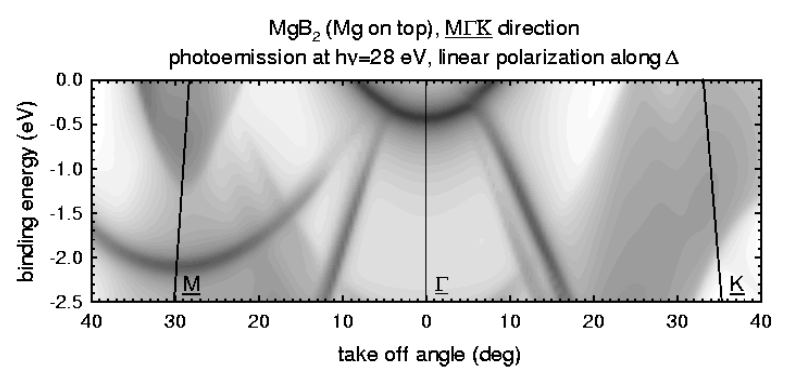

FIG. 4. Theoretical ARPES energy distribution curves taken along $\Sigma$ (left panel) and $\Lambda$ (right panel) symmetry directions for a MgTS. The intensity is measured by the darkness.

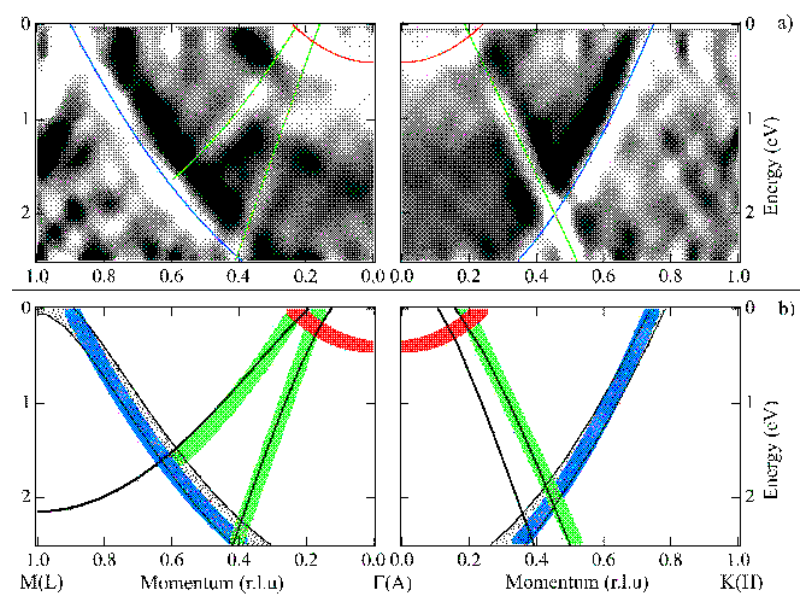

FIG. 5. ((Color), after Uchiyama et al.[1]) Experimental ARPES data (upper panel). The intensity is measured by the brightness obtained from the second derivative of the ARPES spectra. The assignment of one SFS (red) and bulk states $(\pi$ (blue) and $\sigma$ (green)) proposed in Ref. 1 (lower panel).

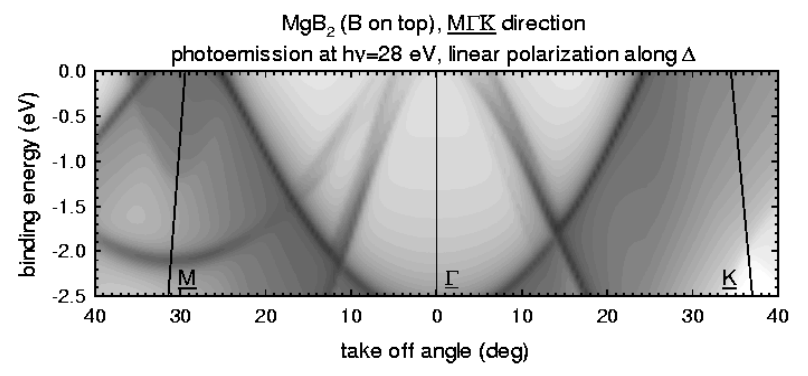

FIG. 6. The same as in Fig. 4 for a BTS.

be observed first in the SFS, because the screening of Coulomb interactions is weaker near the surface rather than in the bulk. Taking into account the perfect agreement of LDA with the ARPES data, we conclude that such many-body effects should be of minor importance. With respect to surface superconductivity to be discussed below, the experimental determination of the electronphonon (el-ph) coupling constant $\lambda$ from the temperature dependence of the ARPES spectra like in Be would be of great interest [12,13.

Now we address possible consequences of the SFS with respect to physical properties of various samples with emphasis on superconductivity and micro-powder samples. Since for typical samples the size in $c$-direction is about $10 \mathrm{~nm}$ only, about one quarter of the volume is affected by the presence of SFS. Since for MgTS the SFS shown in Fig. 1 is derived from orbitals with cylindrical symmetry and there is no contribution from orbitals with $p_{x, y}$ character, a very weak local el-ph interaction is expected. Thus it acts as a bad or non- superconducting layer in the sense of the proximity effect. Due to the interaction with the bulk states the resulting superconductivity near a MgTS will be weakened even for a clean MgTS, at variance with the view put forward in Ref. 3 .

Thus the presence of such SFS might explain the absence of a third upper critical field $H_{c 3}$ [4 and the local suppression of superconductivity in relatively weak external magnetic field slightly above 2 Tesla [5]. This was regarded as evidence for a small value of $H_{c 2}^{c}$, i.e. a big anisotropy $\gamma_{H} \sim 6$ to 9 . Anyhow, this would be in sharp contrast to the anisotropy caused by the Fermi velocities $\gamma_{v}^{\sigma} \sim 6$ to 7 which yields $\gamma_{H}^{\sigma} \sim 4.2$ to 5.5 as an upper limit [14] derived for the strongly interacting subsystem of $\sigma$-holes. [15] Due to the two-band character of $\mathrm{MgB}_{2}$ [15]17] which involves also the weakly interacting nearly isotropic $\pi$-electrons the total $\gamma_{H}$ is further reduced till below 4 to 5 , provided any order parameter anisotropy of the two different gaps can be neglected [17,18]. A moderate $\gamma_{H} \sim 3$ is in accord with available single crystal data. 15

The interplay of superconducting bulk and SFS subsystems considered here contrasts the approach adopted in Ref. 19 where due to symmetry breaking at the surface mixing and pair breaking of $\sigma$-and $\pi$-states in the sense of a two-band model occurs. Notably, the absence or presence of superconducting SFS may affect the surface pinning. Bulk vortices will be attracted or repelled, respectively. This might explain the strong variations of pinning properties after applying pressure, which might affect the interfaces of the grains. Furthermore the presence of surface superconductivity on SFS will affect the tunneling data. In the context of frequently considered $3 \mathrm{D}$ multi-band models for $\mathrm{MgB}_{2}$ it is important to make a correct assignment of the observed superconducting gaps. In particular, in view of the ARPES interpretation we suggested above, the gap at about $5 \mathrm{meV}$ observed by high-resolution (angle integrated) photoemission spectroscopy 20] might be ascribed to superconductivity on SFS.

Up to now two-dimensional (2D) electrons in metals were observed only in artificial layered structures [21]. Let us consider in short the new physics which can be gained by studying generic $2 \mathrm{D}$ electrons at the surface of a 3D superconductor. As most interesting we consider the possibility for enhancement of the superconductivity of these $2 \mathrm{D}$ electrons due to increased local DOS or soft- 
ening of local phonon modes at the surface. In this case in the framework of Ginzburg-Landau theory, for example, we have to introduce a $2 \mathrm{D}$ superconducting order parameter $\Psi_{2 D}$ in parallel to the bulk one $\Psi_{3 D}$; additionally the phenomenology could be complicated by necessity of taking into account pairing in different and weakly interacting bands. As a whole the surface enhancement of superconductivity could remind the twinning plane superconductivity observed in Sn and Nb. [22] In this case the dependence of magnetization as a function of temperature and magnetic field can be similar to the fluctuation conductivity of the bulk. Roughly speaking, due to the proximity effect $2 \mathrm{D}$ conductivity can create a "frozen" 3D fluctuation. A systematic study of fluctuation magnetism in powdered samples with different grain sizes can give important hints in this direction. It will be very informative to perform investigation of fluctuation magnetism [23 and to determine the mass anisotropy of fluctuation Cooper pairs using $\mathrm{MgB}_{2}$ microcrystals sintered under high pressure [24].

However it is more plausible that the influence of the surface is to reduce the local superconductivity: we have to take into account despite the possible $\mathrm{Mg}$ or B terminations of the crystal, also the adsorption of various impurities etc [3]. In this case the influence of the surface can be traced out in the vortex pinning in the superconducting state. Indeed, strong asymmetric hysteresis loops were observed after the grinding of a bulk sample into fine powder 25]. Surface pinning was reported for a powder sample pressed into a pellet without sintering. [26] Both possibilities, enhancement and suppression of superconductivity yield additional surface pinning. Finally, we argue that $2 \mathrm{D}$ electronic states can give an important contribution to the current dependent correction of the work function of the superconducting metal, the so called Bernoulli potential.

We have shown that the available ARPES data can be well described by the LDA. In particular, there is clear evidence for the presence of at least two SFS. Since the SFS depend very sensitively on the nature of the terminated layer, comparing our calculated intensities with the measured ones, we suggest that the studied single crystal contained both types of terminated layers. Therefore the investigation of especially prepared surfaces with only one predominant surface layer is of interest. In this context the theoretical study of steps between $\mathrm{B}$ and $\mathrm{Mg}$ terminated layers would be interesting. Since the SFS are generated by the large gapped region in the BZ, similar phenomena might be expected for other layered superconductors such as $\mathrm{NbSe}_{2}$ and even heavy fermion compounds $\mathrm{URu}_{2} \mathrm{Si}_{2}$ or borocarbides [27] where also no $H_{c 3}$ has been observed. Taking into account all those possibilities mentioned above one can expect that comprehensive studies of $\mathrm{MgB}_{2}$ could generate interesting physics of the surface/interface of superconductors.

V.D.P.S. is grateful to A.N. Yaresko and S.V. Halilov who respectively provided the LMTO and layer-KKR codes used in the present study. We are indepted to S. Tajima for providing us with the ARPES and other single crystal data prior to publication. We thank S. Tajima, H. Uchiyama, A. Kordyuk, G. Fuchs, S. Shulga, M. Richter, G. Paasch, J. Fink, H. Eschrig, C. Laubschat, and H. Rosner for discussions. V.D.P.S. was supported by the Deutsche Forschungsgemeinschaft (project La-655/7-1).

[1] H. Uchiyama, K.M. Shen, S. Lee, A. Damascelli, D.H. Lu, D.L. Feng, Z.-X. Shen, and S. Tajima, Phys. Rev. Lett. submitted, cond-mat/0111152.

[2] V.M. Silkin, el al., Phys. Rev. B 64, 172512 (2001).

[3] In Gee Kim el al., Phys. Rev. B 64, 020508 (2001).

[4] O.F. Lima, in Studies of High Temperature Superconductors" (Ed. Narlikar), Nova Science, N.Y. 38 (2001).

[5] S.L. Budko el al., cond-mat/0106577.

[6] F. Simon, el al., Phys. Rev. Lett. 87 (2001) 047002.

[7] G. Papavassiliou el al., cond-mat/0107511.

[8] S.V. Halilov et al., J. Phys.: Cond. Matter 5, 3859 (1993).

[9] U. von Barth and L. Hedin, J. Phys. C 12, 1629 (1972).

[10] A. Alexandrov, cond-mat/0104413 (2001).

[11] E. Cappelluti el al.,cond-mat/0104413 (2001).

[12] T. Balasubramanian el al., Phys. Rev. B, 57 R6866 (1998).

[13] M. Hengsberger el al., Phys. Rev. Lett. 83592 (1999).

[14] E. Langmann, Physica C, 173, 347 (1991).

[15] G. Fuchs, S.-L. Drechsler, S.V. Shulga, A. Handstein, K. Nenkov, V. Narozhnyi, K.-H. Müller, and H. Rosner, in Studies of High Temperature Superconductors" (Ed. Narlikar), Nova Science, N.Y. 41 (2001), in press.

[16] S.V. Shulga, S.-L. Drechsler, H. Eschrig, H. Rosner, and W. Pickett, cond-mat/0103154.

[17] H. Rosner, J.M. An, W. Ku, M.D. Johannes, R.T. Scalettar, W. Pickett, S.V. Shulga, S.-L. Drechsler, H. Eschrig, W. Weber, and A.G. Eguilez in Studies of High Temperature Superconductors" (Ed. Narlikar), Nova Science, N.Y. 38 (2001), in press.

[18] H.J. Choi el al., cond-mat/0111183.

[19] E. Bascones and F. Guinea, Phys. Rev. B (2001).

[20] T. Takahashi el al., Phys. Rev. Lett. 86, 4915 (2001).

[21] T.C. Hsieh el al., Phys. Rev. Lett. 55, 2483 (1985).

[22] I.N. Khlyustikov and A.I. Buzdin, Adv. Phys. 36, 271 (1987) and references therein. We note that there is a sizable body of papers devoted to various aspects of surface superconductivity. However, to the best of our knowledge there is no systematic study for the interplay of bulk and surface superconductivity with explicit inclusion of SFS.

[23] A. Lascialfari, T. Mishonov, A. Rigamonti, P. Tedesco, and A. Varlamov, cond-mat/0109404.

[24] N.E. Filonenko, el al.,Technical Physics, Translated from Dokladi Akademii Nauk SSSR, 175, 1266 (1967).

[25] M. Pissas el al., cond-mat/0108153.

[26] M.J. Qin el al., cond-mat/0108172.

[27] K. Winzer, private communication. 